\title{
Psychometric properties of the Valuing Questionnaire in a Spaniard sample and factorial equivalence with a Colombian sample
}

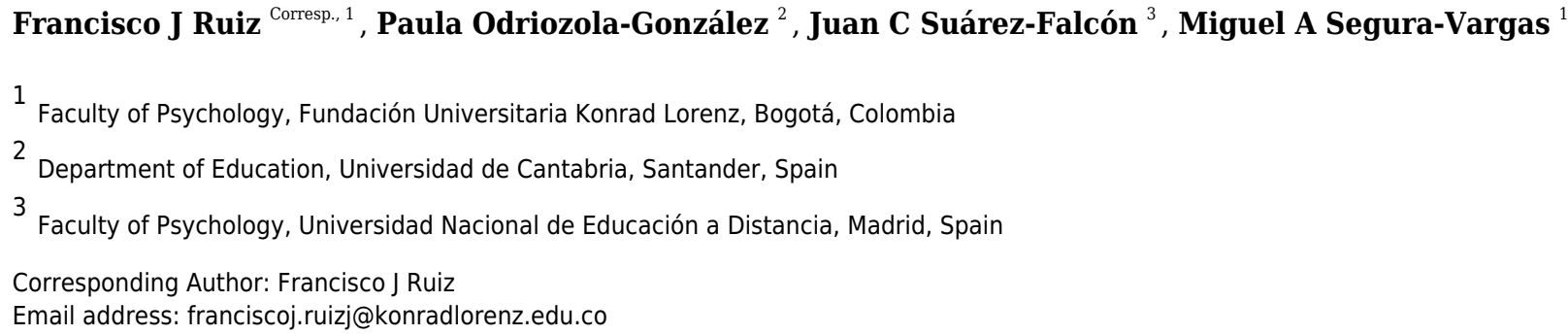

Background. The Valuing Questionnaire (VQ) is considered as one of the most psychometrically robust instruments to measure valued living according to the acceptance and commitment therapy model. It consists of 10 items that are responded to on a 7-point Likert-type scale and has two factors: Progression and Obstruction. The Spanish version of the VQ showed good psychometric properties in Colombian samples. However, there is no evidence of the psychometric properties of the VQ in Spaniard samples. This study aims to analyze the validity of the VQ in a large Spaniard sample and analyze the measurement invariance with a similar Colombian sample. Method. The VQ was administered to a Spaniard sample of 846 adult participants from general online population. Cronbach's alpha and McDonald's omega were computed to analyze the internal consistency of the VQ. The fit of the VQ's two-factor model was tested through a confirmatory factor analysis with a robust maximum likelihood (MLR) estimation method. Afterward, we analyzed the measurement invariance across countries and gender. Convergent construct validity was analyzed with a package of questionnaires that evaluated experiential avoidance (Acceptance and Action Questionnaire-II, AAQ-II, emotional symptoms (Depression Anxiety and Stress Scale-21, DASS-21), life satisfaction (Satisfaction with Life Scale, SWLS), and cognitive fusion (Cognitive Fusion Questionnaire, CFQ). Results. The internal consistency across samples was adequate (alphas and omegas were .85 for VQ-Progress and .84 for VQ-Obstruction). The two-factor model obtained a good fit to the data (RMSEA $=0.073$, $90 \% \mathrm{Cl}[0.063,0.083], \mathrm{CFI}=0.98, \mathrm{NNFI}=0.97$, and SRMR $=0.053)$. The VQ showed strict invariance across countries and gender and showed theoretically coherent correlations with emotional symptoms, life satisfaction, experiential avoidance, and cognitive fusion. In conclusion, the Spanish version of the VQ demonstrated good psychometric properties in a large Spaniard sample. 


\section{Psychometric properties of the Valuing Questionnaire}

3 in a Spaniard sample and factorial equivalence with a

4 Colombian sample

5

6

7 8 9

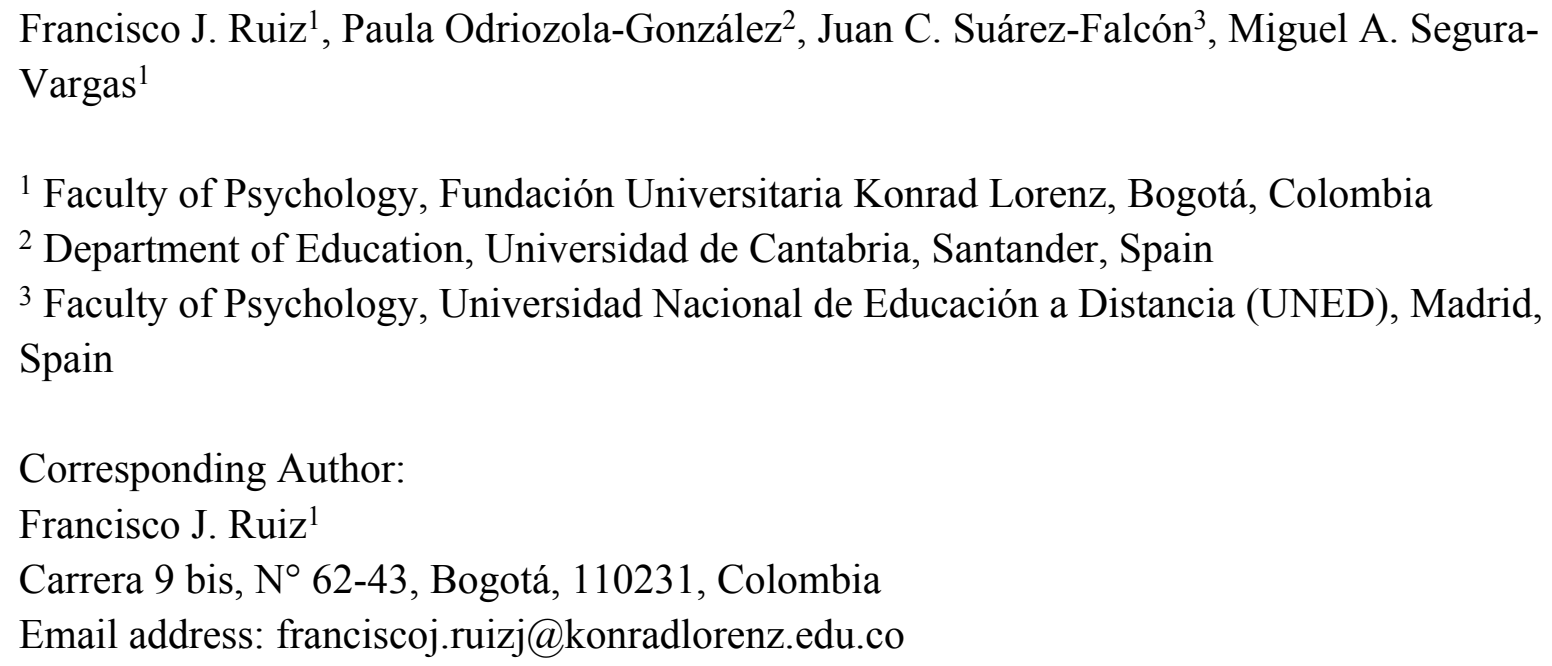




\section{Abstract}

29 Background. The Valuing Questionnaire (VQ) is considered as one of the most

30 psychometrically robust instruments to measure valued living according to the acceptance and

31 commitment therapy model. It consists of 10 items that are responded to on a 7-point Likert-type

32 scale and has two factors: Progression and Obstruction. The Spanish version of the VQ showed

33 good psychometric properties in Colombian samples. However, there is no evidence of the

34 psychometric properties of the VQ in Spaniard samples. This study aims to analyze the validity

35 of the VQ in a large Spaniard sample and analyze the measurement invariance with a similar

36 Colombian sample.

37 Method. The VQ was administered to a Spaniard sample of 846 adult participants from general

38 online population. Cronbach's alpha and McDonald's omega were computed to analyze the

39 internal consistency of the VQ. The fit of the VQ's two-factor model was tested through a

40 confirmatory factor analysis with a robust maximum likelihood (MLR) estimation method.

41 Afterward, we analyzed the measurement invariance across countries and gender. Convergent

42 construct validity was analyzed with a package of questionnaires that evaluated experiential

43 avoidance (Acceptance and Action Questionnaire-II, AAQ-II, emotional symptoms (Depression

44 Anxiety and Stress Scale-21, DASS-21), life satisfaction (Satisfaction with Life Scale, SWLS),

45 and cognitive fusion (Cognitive Fusion Questionnaire, CFQ).

46 Results. The internal consistency across samples was adequate (alphas and omegas were .85 for

47 VQ-Progress and .84 for VQ-Obstruction). The two-factor model obtained a good fit to the data

$48(\mathrm{RMSEA}=0.073,90 \% \mathrm{CI}[0.063,0.083], \mathrm{CFI}=0.98, \mathrm{NNFI}=0.97$, and $\mathrm{SRMR}=0.053)$. The

49 VQ showed strict invariance across countries and gender and showed theoretically coherent

50 correlations with emotional symptoms, life satisfaction, experiential avoidance, and cognitive 
51 fusion. In conclusion, the Spanish version of the VQ demonstrated good psychometric properties

52 in a large Spaniard sample. 


\section{Introduction}

54

55

56

57

58

59

60

61

62

63

64

65

66

67

68

69

70

71

72

73

74 intrinsically reinforced dynamic patterns of activity that lead the individual symbolically closer

75

Acceptance and commitment therapy (ACT; Hayes, Strosahl, \& Wilson, 1999) is an empirically based psychological intervention (Gloster, Walder, Levin, Twohig, \& Karekla, 2020) that emphasizes the role of psychological flexibility on mental health and behavioral effectiveness. Psychological flexibility is usually defined in middle-level terms, which are higher-level functional abstractions that serve as shortcuts for applying basic principles to complex applied settings (Vilardaga, Hayes, Levin, \& Muto, 2009). One of the most widely used definitions of psychological flexibility states that is "the ability to contact the present moment more fully as a conscious human being, and to change or persist in behavior when doing so serves valued ends" (Hayes, Luoma, Bond, Masuda, \& Lillis, 2006, p. 7). As such, psychological flexibility comprises six core therapeutic processes: cognitive defusion (noticing thinking in flight in a detached and non-judgmental way), acceptance (being open to experience unpleasant private experiences), contact with the present moment (attention flexibility), self-as-context (noticing ongoing behavior from an inclusive and transcendent perspective), values (verbally constructed positive reinforcers), and committed action (build and broaden values-based behavioral patterns). Values is a crucial process in the psychological flexibility model because they provide direction and meaning to the individual's behavior.

More specifically, values are conceptualized as verbally constructed positive reinforcers that are at the top of a hierarchy of reinforcers, including goals and more tangible reinforcers (Barnes-Holmes, Barnes-Holmes, McHugh, \& Hayes, 2004; Gil-Luciano, Calderón-Hurtado, Tovar, Sebastián, \& Ruiz, 2019; Luciano, Valdivia-Salas, \& Ruiz, 2012). Values entail to his/her values (Wilson, 2009). The specific activities that would permit advancing towards 
76 own values frequently vary over time and across situations. For instance, a valued action for an

77 undergraduate whose exams are getting close would probably be studying, whereas on holidays,

78 valued actions might be related to activities involving social relationships.

79 According to the ACT model, values are related to joy, meaning, and suffering in two

80 main ways (Ruiz, 2020). Firstly, thoughts and actions symbolically related to these hierarchical

81 positive reinforcers acquire intense appetitive functions (Gil, Luciano, Ruiz, \& Valdivia-Salas,

82 2012; Gil-Luciano et al., 2019). For instance, when valuing a romantic relationship characterized

83 by sharing and transparency, telling a traumatic experience will acquire appetitive functions that

84 might undermine the aversive functions actualized when sharing that experience. Similarly,

85 thoughts and actions symbolically related in opposition to these hierarchical positive reinforcers

86 (i.e., values) will acquire intense aversive functions (Gil-Luciano et al., 2019; Ruiz, Luciano,

87 Flórez, Suárez-Falcón, \& Cardona-Betancourt, 2020). In summary, values and suffering usually

88 become the two sides of the same coin (Wilson, 2009). Secondly, suffering is exacerbated as a

89 consequence of displaying an inflexible pattern of behavior characterized by the entanglement

90 with unpleasant private experiences and engagement in experiential avoidance strategies (Hayes

91 et al., 2006). This behavioral inflexibility reduces the frequency of valued actions, leading to

92 experiencing more aversive thoughts and emotions in opposition to values (Ruiz et al., 2020).

93 Given the central role of values in the ACT model, numerous self-report measures of this

94 process have been developed in the last decade, and three recent systematic reviews have been

95 published (Barrett, O'Connor, \& McHugh, 2019; Reilly et al., 2019; Serowik et al., 2018). Some

96 of the most used instruments of values are the Valued Living Questionnaire (VLQ; Wilson,

97 Sandoz, Kitchens, \& Roberts, 2010), the Bull's-Eye Values Survey (BEVS; Lundgren, Luoma,

98 Dahl, Strosahl, \& Melin, 2012), the Engaged Living Scale (ELS; Trompetter et al., 2013), and 
99 the Valuing Questionnaire (VQ; Smout, Davies, Burns, \& Christie, 2014). Values self-reports

100 can be grouped according to their procedure. Some questionnaires rate to what extent

101 participants value pre-established different life domains and the consistency of their behavior

102 according to their values (e.g., VLQ, BVES). Other instruments measure overall valued living

103 without specifying pre-established life domains (ELS and VQ). Values instruments that explore

104 life domains are more informative than general measures, but they have the limitation that are

105 time-consuming. General values measures are often short and easier to score and interpret.

106 According to the systematic reviews mentioned before, values instruments vary in their

107 psychometric quality. Two of these reviews indicate that the VQ is probably the most

108 psychometrically robust instrument (Barret et al., 2019; Reilly et al., 2019). In the original study

109 of the VQ, Smout et al. (2014) asked 630 undergraduates to grade a pool of 70 items, which

110 representativeness was previously rated by eight ACT experts. By using a Rating Scale Model

111 and a Partial Credit Model, the authors retained 10 of the 70 original items. These 10 items were

112 administered to a second sample. The final version of the VQ showed a two-factor structure with

113 the following subscales: Progress (i.e., enactment of values, including clear awareness of what is

114 personally meaningful and perseverance) and Obstruction (i.e., disruption of valued living due to

115 avoidance of unwanted experience and distraction from values). The internal consistency of both

116 subscales was good (from .81 to .87 for Progress and from .79 to .87 for Obstruction). The VQ-

117 Progress scores showed strong correlations with positive affect, well-being, life satisfaction, and

118 mindfulness skills. Conversely, scores on the VQ-Obstruction showed strong positive

119 correlations with emotional symptoms, negative affect, and experiential avoidance.

120 Across studies, the VQ has shown excellent psychometric properties, with good internal

121 consistency, structural validity, and a higher sensitivity to treatment than other values measures 
122 (Barrett et al., 2019; Reilly et al., 2019). The convergent construct validity of the VQ has been

123 supported in subsequent studies (Carvalho, Palmeira, Pinto-Gouveia, Gillanders, \& Castilho,

124 2018; Rickardsson et al., 2019; Ruiz, Suárez-Falcón, Segura-Vargas, \& Gil-Luciano, 2021).

125 Indeed, two systematic reviews indicated that the VQ is probably the most psychometrically

126 robust instrument of values according to the ACT model (i.e., Barrett et al., 2019; Reilly et al., 127 2019).

128 Unfortunately, the psychometric properties of the VQ have not been widely studied 129 across different cultures and countries. To our best knowledge, there are only three validation 130 studies of the VQ in other languages. Two studies analyzed the validity of the VQ in the context 131 of chronic pain in Portuguese and Swedish (Carvalho et al., 2018; Rikardsson et al., 2019). In the 132 remaining study, Ruiz et al. (2021) administered the Spanish version of the VQ to three 133 Colombian samples. The VQ showed good internal consistency across samples, and the two134 factor model obtained a good fit to the data. Additionally, scalar measurement invariance was 135 found across clinical and nonclinical samples and gender.

136 To our best knowledge, no studies have been conducted in Spain regarding the 137 psychometric properties of the VQ. This could hinder research aimed to study the process of 138 values from the ACT standpoint with Spaniard samples. Additionally, no studies have explored 139 the measurement invariance across cultures. Therefore, this study aimed to analyze the validity 140 of the VQ with a Spaniard sample and the factorial equivalence with a Colombian sample. We 141 expected that the VQ would show good internal consistency and a good fit of the two-factor 142 structure in the Spaniard sample. Additionally, we expected to find measurement invariance 143 across countries and gender. Likewise, we expected to find positive correlations between VQ-

144 Progress and life satisfaction and negative correlations with emotional symptoms, experiential 
145 avoidance, and cognitive fusion. An inverse pattern of correlations was expected between VQ-

146 Obstruction and the remaining constructs.

147

\section{Materials \& Methods}

\section{Procedure}

According to Ato, López-García, and Benavente (2013), an instrumental design was implemented by analyzing the psychometric properties of the VQ and its measurement invariance across gender and countries. The procedures implemented in this research were approved by the Bioethics Committee of Fundación Universitaria Konrad Lorenz (2016-021B). Informed written consents were obtained from all participants in this study.

Participants in both samples responded to an anonymous online survey distributed through social media (e.g., institutional webpages, Facebook, etc.) through a snowball sampling procedure. Specifically, the researchers asked their contacts and participants to share the publication of the survey to reach more people. The research publication was not posted in specific groups (e.g., students' or mental health groups).

Participants first provided informed consent by accepting the conditions described at the beginning of the survey that included that they were adults (i.e., the only exclusion criterion to participate being younger than 18 years). Afterward, the participants in Sample 1 completed the instruments presented above. Participants in Sample 2 responded to a similar survey, but only the VQ data are relevant for this study. Their respective instructions preceded each questionnaire. Participants were also emphasized that the survey was anonymous and that they could stop their participation anytime they wanted. The median time for the completion of surveys was approximately 15 minutes. When data collection was finished, global reports of the results of the 
168 surveys were sent to the participants who indicated that they were interested in them. Participants 169 were not compensated for their participation.

170 The survey conducted to recruit Sample 1 was available for one year until at least 200

171 participants of both genders responded. As we planned to analyze measurement invariance

172 across gender, this number was established because it is the minimum suggested, as a rule of

173 thumb, for conducting confirmatory factor analyses (Kline, 2016).

\section{Participants}

Sample 1. This sample consisted of 846 Spanish participants (75.7\% females) with an age

176

177

178

179

180

181

182

183

184

185

186

187

188

189

190

range between 18 and $72(M=35.40, S D=11.39)$. The participants' relative education level was

$0.1 \%$ no studies, $33 \%$ primary studies (i.e., compulsory education) or mid-level study graduates (i.e., high school or vocational training), and 65.6\% were undergraduates or college graduates (1.3\% did not indicate the educational level). Almost half of the participants $(44.6 \%)$ reported having received psychological or psychiatric treatment in the past, but only $12.8 \%$ were currently in treatment. Also, $12.9 \%$ of participants reported using psychotropic medication.

Sample 2. This sample consisted of 724 Colombian participants (74.4\% females) whose ages ranged between 18 and $88(M=26.11, S D=8.93)$. The participants' relative education level was $17.8 \%$ primary studies or mid-level study, and $82.2 \%$ were undergraduates or college graduates. Forty-five percent reported having received psychological or psychiatric treatment at some time, but only $8.4 \%$ were currently in treatment. Also, $5.4 \%$ of participants reported using psychotropic medication.

\section{Instruments}

Valuing Questionnaire (VQ; Smout et al., 2014; Spanish translation by Ruiz et al., 2021). The VQ is a 10-item, 7-point Likert (6= completely true; $0=$ not at all true $)$, self-report 
191 instrument designed to assess general valued living over the last week. It assesses valued living

192 in everyday language without referring to specific life domains. The VQ has two 5-item factors:

193 Progress (i.e., enactment of values, including clear awareness of what is personally meaningful

194 and perseverance) and Obstruction (i.e., disruption of valued living due to avoidance of

195 unwanted experiences and distraction from values). The Spanish version of the VQ has shown

196 good psychometric properties in Colombian samples, with alphas of .83 and .82 for Progress and

197 Obstruction, respectively.Acceptance and Action Questionnaire - II (AAQ-II; Bond et al., 2011;

198 Spanish version by Ruiz, Langer, Luciano, Cangas, \& Beltrán, 2013). The AAQ-II consists of 7

199 items that measure experiential avoidance on a 7-point Likert-type scale $(7=$ always true; $1=$

200 never true). The Spanish version of the AAQ-II has shown good psychometric properties (mean

201 alpha of .88) and a one-factor structure in Spanish samples (Ruiz et al., 2013). In this study, the

202 AAQ-II showed an alpha of .91. Strong positive correlations were expected between AAQ-II and

203 VQ-Obstruction, whereas at least medium negative correlations were expected with VQ-

204 Progress.

205 Cognitive Fusion Questionnaire (Gillanders et al., 2014; Spanish version by Ruiz,

206 Suárez-Falcón, Riaño-Hernández, \& Gillanders, 2017). The CFQ is a 7-item, 7-point Likert-type

207 scale $(7=$ always true $; 1=$ never true $)$ of general cognitive fusion. The Spanish version by Ruiz,

208 Suárez-Falcón, et al. (2017) has shown excellent internal consistency (alpha of .92) and a one-

209 factor structure. In this study, the AAQ-II showed an alpha of .93. As with the AAQ-II, strong

210 positive correlations were expected between CFQ and VQ-Obstruction, whereas at least medium

211 negative correlations were expected with VQ-Progress.

212 Depression, Anxiety, and Stress Scales - 21 (DASS-21; Lovibond \& Lovibond, 1995;

213 Spanish version by Daza, Novy, Stanley, \& Averill, 2002). The DASS-21 is a 21-item, 4-point 
214 Likert-type scale ( 3 = applied to me very much, or most of the time; $0=$ did not apply to me at 215 all) consisting of sentences describing negative emotional states: Depression, Anxiety, and 216 Stress. The Spanish version of the DASS-21 has shown good internal consistency for all the 217 subscales and a hierarchical factor structure with a second-order factor (Ruiz, García-Martín, 218 Suárez-Falcón, \& Odriozola-González, 2017). In this study, the DASS-21 obtained alphas of .95, $219.92, .87$, and .86 for the total scale, Depression, Anxiety, and Stress, respectively. Strong positive 220 correlations were expected between the DASS-21 and VQ-Obstruction, whereas at least medium

221

222

223 224 225 226 227 228 229 230 231 232 233 234 235 236 negative correlations were expected with VQ-Progress.

Satisfaction with Life Survey (SWLS; Diener et al., 1985; Spanish translation by Atienza et al., 2000). The SWLS is a 5-item survey that evaluates self-perceived well-being through a 7point Likert-type scale $(7=$ strongly agree; 1 = strongly disagree $)$. Ruiz et al. (2019) found that the Spanish version of the SWLS showed good psychometric properties and convergent validity in a Spaniard sample. In this study, the SWLS showed an alpha of .89. Strong positive correlations were expected between the SWLS and VQ-Progress, and medium negative correlations were expected with VQ-Obstruction.

\section{Statistical and Psychometric Analysis}

Firstly, we analyzed the fit of the two-factor model of the VQ by computing confirmatory factor analyses (CFAs) with Sample 1 (i.e., the Spaniard sample). In so doing, we followed the procedure used in the Spanish validation of the VQ in Colombia (Ruiz et al., 2021). As previous CFAs of the VQ have shown a method effect in Items 5 and 7 responses (Smout et al., 2014), we decided to compare the fit of the two-factor model where the error terms between these items were allowed to correlate versus the two-factor model with no error correlations. The software LISRELC (version 8.71, Jöreskog \& Sörbom, 1999) was used to conduct the CFAs. No missing 
237 values were found due to the collection method (i.e., online survey). Given the lack of

238 multivariate normality in the data (multivariate Mardias' test of skewness and kurtosis $=610.954$;

$239 \mathrm{p}<.001$ ), we selected the robust maximum likelihood (MLR) estimation method with the

240 covariance matrix and the asymptotic variance-covariance matrix. The robust estimation

241 methods in structural equation modeling are known for overcoming to a great extent the problem

242 of the presence of outliers (Yuan \& Zong, 2013). Even so, we have also used z-scores and

243 Mahalanobis distance and obtained virtually the same results with or without the few identified

244 outliers.

245 We computed the Satorra-Bentler chi-square test and the following goodness-of-fit

246 indexes for the two-factor model: (a) the comparative fit index (CFI), (b) the non-normed fit

247 index (NNFI), (c) the root mean square error of approximation (RMSEA), (d) the parsimony

248 normed fit index (PNFI), and (e) the standardized root mean square residual (SRMR). According

249 to authoritative guidelines (e.g., Hu \& Bentler, 1999), CFI and NNFI values above .90 represent

250 acceptable models, and above .95 indicate a good fit to the data. Regarding the RMSEA, values

251 below 0.08 represent an acceptable fit, and values below 0.05 constitute a good fit (Browne \&

252 Cudeck, 1992, 1993; Hooper, Coughlan, \& Mullen, 2008). Concerning the SRMR, values of

2530.08 represent a good fit, and values below 0.05 represent a very good fit to the data.

254 Furthermore, higher PNFI values indicate a more parsimonious model. Lastly, we also calculated 255 the p-value for test of close fit (PCLOSE), which computes a one-sided test of the null 256 hypothesis that RMSEA is equal or lower than 0.05 .

257 Secondly, we analyzed construct reliability and evidence of convergent and discriminant

258 validity of the measurement model following the suggestions by Brown (2015) and Fornell and

259 Larcker (1981). The composite reliability coefficient (CR) was computed to analyze construct 
260 reliability. CR values higher than 0.70 can be considered as high construct reliability and 261 adequate internal consistency. The convergent validity of the measurement model was analyzed

262 according to three criteria: (a) factor loadings should be statistically significant (standardized

263 loadings estimates should be 0.40 or higher), (b) CR should be higher than 0.70 , and (c) the 264 average variance extracted (AVE) should be $\geq 0.50$ for each subscale of the VQ. Lastly, 265 discriminant validity was estimated according to the following criteria: (a) inter-construct 266 correlations should be lower than 0.80 , and (b) the square root of AVE ( $\sqrt{ } A V E)$ of both factors of 267 the VQ should be greater than the inter-construct correlations with any other factor.

268 Thirdly, we analyzed the measurement invariance across countries (i.e., Spain and 269 Colombia) and gender. In so doing, we followed also the procedure used by Ruiz et al. (2021) in 270 the Spanish validation of the VQ in Colombia. Specifically, we adopted the guidelines proposed 271 by Jöreskog (2005) and Millsap and Yun-Tein (2004). Through additional CFAs, we tested 272 metric, scalar, and strict invariances by analyzing whether the item factor loadings, items 273 intercepts, and the variance of error of the items were invariant across countries and gender. The 274 relative fit of four progressively more restrictive models was compared. Firstly, the multiple275 group baseline model allowed the unstandardized factor loadings to vary across groups.

276 Conversely, the pattern of item-factor loadings and the number of factors were the same across 277 groups (configural invariance). Secondly, the metric invariance model was nested within the 278 previous model and placed equality of factor loadings across groups (i.e., weak factorial 279 invariance). Thirdly, the scalar invariance model was nested within the metric invariance model 280 and confined the factor loadings and the items intercepts to be the same across groups (i.e., 281 strong factorial invariance). Lastly, the strict invariance model was nested within the scalar 282 invariance and assumed the variance of errors (i.e., indicator residuals) to be equal across groups. 
283 Following Kline (2005) indications, we did not place equality constraints on estimates of the 284 factor variances because they differ across groups even when the indicators are quantifying the 285 same construct in an equivalent manner. For the model comparison, we weighed the CFI, NNFI, 286 and RMSEA indices between nested models. We chose the more constrained model (i.e., second 287 model versus the first model, and third model versus the second model) following the criteria 288 advocated by Cheung and Rensvold (2002) and Chen (2007): (a) the difference in RMSEA 289 ( $\triangle$ RMSEA) was lower than .01; (b) the differences in NNFI ( $\triangle \mathrm{NNFI)}$ and CFI $(\Delta \mathrm{CFI})$ were 290 equal to or higher than -.01 .

291 Fourthly, we analyzed the internal consistency of the VQ in Sample 1 with the MBESS 292 package in R (Kelley \& Lai, 2012; Kelley \& Pornprasertmanit, 2016). We computed corrected 293 item-total correlations to identify items that should be removed because of showing a low 294 discrimination item index (i.e., values below .30). Then, we calculated coefficient alphas and 295 McDonald's omegas and provided percentile bootstrap 95\% confidence intervals (CI). According 296 to Nunnally and Bernstein (1994), values higher than .70 were considered acceptable, whereas 297 above .80 were considered good. To determine what label would apply to the VQ subscales, we 298 observed the 95\% CI. Fifthly, we calculated Pearson correlations between the VQ and the 299 remaining scales to assess evidence based on relationships with other variables using SPSS 25C.

300 Correlations were interpreted according to the guidelines provided by Lenhard and Lenhard 301 (2016): small correlation between .10 and .20, medium between .21 and .36, and strong 302 correlations $>.36$. Lastly, we calculated descriptive data and explored differences in VQ scores 303 across country and gender by computing independent sample t-tests. 
306 Validity evidence based on internal structure

307

308

309

310

311

312

313

314

315

316

317

318

319

320

321

322

323

324

325

326

327

328

\section{Dimensionality}

Table 1 shows the results of the CFAs conducted in Sample 1. The fit of the two-factor model of the VQ was good according to the values of CFI (.98), NNFI (.97), and SRMR (0.053).

The RMSEA value was 0.073 , which indicates an acceptable fit. However, the test of close fit (PCLOSE) showed that the RMSEA value was significantly higher than $0.05(\mathrm{p}<.01)$. Thus, overall, the two-factor model showed an acceptable fit according to this goodness-of-fit index. The alternative two-factor model with correlated error terms in Items 5 and 7 did not significantly improve the fit. In conclusion, we chose the two-factor model due to its greater parsimony according to the PNFI (see Table 1). This was also the factor model selected in Ruiz et al. (2021) with Colombian participants. Figure 1 depicts the results of the completely standardized solution of the two-factor model in Sample 1.

INSERT TABLE 1 ABOUT HERE

INSERT FIGURE 1 ABOUT HERE

\section{Construct Reliability and Convergent and Discriminant Validity of the Measurement}

\section{Model}

The VQ showed high construct reliability because the CR values were higher than 0.70 for both VQ-Progression (0.85) and VQ-Obstruction (0.84). The convergent validity of the VQ was also supported. First, all factor loadings were statistically significant and higher than 0.40 (with six items exceeding the ideal cutoff of 0.70). Second, the AVE values of both VQ-Progress (0.54) and VQ-Obstruction (0.52) were higher than 0.50. Lastly, the CR values of both constructs were very high. The VQ also obtained indicators of appropriate discriminant validity. First, the inter-construct correlation was -0.60 , which is lower in absolute value than 0.80 . 
329 Second, the square roots of AVE were greater than the inter-construct correlation of this latent

330 variable with any of the factors (VQ-Progress: $\sqrt{ } A V E=0.73$; VQ-Obstruction $=(\sqrt{ } A V E=0.72)$.

331 Measurement invariance

332 Table 2 presents the results of the metric, scalar, and strict invariance analyses. Parameter

333 invariance was supported at all levels across countries (Spain and Colombia) and gender because

334 there were no differences higher than 0.01 in the RMSEA, CFI, and NNFI favoring the models

335 with lower constraints. Thus, measurement invariance across countries and gender was

336 demonstrated.

337

338

339

340

341

342

343

344

345

346

347

348

349

350

351

\section{INSERT TABLE 2 ABOUT HERE}

\section{Psychometric quality of the items}

Table 3 shows that all corrected item-total correlations of the VQ in Sample 1 were high. For VQ-Progress, they ranged from .52 to .71 in Sample 1, whereas for VQ-Obstruction from .51 to .70. Cronbach's alpha coefficients were adequate for both subscales (VQ-Progress $=.85,95 \%$

CI $[.83,86]$; VQ-Obstruction $=.84,95 \%$ CI $[.82, .86])$. The values of McDonald's omega coefficients were virtually the same (VQ-Progress $=.85,95 \%$ CI $[.83,87]$; VQ-Obstruction $=$ $.84,95 \%$ CI $[.82, .86])$.

\section{INSERT TABLE 3 ABOUT HERE}

\section{Validity evidence based on relationships with other variables}

Table 4 presents the Pearson correlations between the VQ and the assessed constructs.

Overall, the VQ showed correlations in the expected directions and size. Specifically, VQ-

Progress showed strong negative correlations with experiential avoidance and cognitive fusion.

Medium-size correlations were found between VQ-Progress and emotional symptoms measured with the DASS-21. Lastly, VQ-Progress correlated positively and strongly with life satisfaction. 
352 As expected, VQ-Obstruction obtained the opposite pattern of correlations. Specifically, VQ-

353 Obstruction showed strong and positive correlations with experiential avoidance, cognitive

354 fusion, and emotional symptoms. VQ-Obstruction correlated negatively and strongly with life 355 satisfaction.

356

357

358

359

360

361

362

363

364

365

366

367

368

369

370

371

372

373 374 characteristics.

\section{INSERT TABLE 4 ABOUT HERE}

\section{Scores on the VQ across countries and gender}

No statistically significant differences were found on VQ scores across countries on VQProgress (Spain: $M=19.08, S D=6.08$; Colombia: $M=19.50, S D=6.43 ; t(1568)=-1.35, p=$ .18 ) and VQ-Obstruction (Spain: $M=11.31, S D=6.63$; Colombia: $M=11.70, S D=6.88$; $t(1568)=-1.16, p=.25)$. Regarding gender in Spaniard participants (i.e., Sample 1), there were no statistically significant differences neither for VQ-Progress (Men: $M=18.72, S D=6.13$; Women: $M=19.14, S D=6.08 ; t(811)=-0.80, p=.43)$ or VQ-Obstruction (Men: $M=11.14, S D$ $=6.60 ;$ Women: $M=11.39, S D=6.69 ; t(811)=-0.43, p=.67)$.

\section{Discussion}

The VQ is one of the most widely used and psychometrically robust measures of valued living according to the ACT model (e.g., Barrett et al., 2019; Reilly et al., 2019; Serowik et al., 2018). Although there is a Spanish version of the VQ (Ruiz et al., 2021), no studies have analyzed its psychometric properties in Spaniard samples. The current study aimed to fill this gap by analyzing the psychometric properties and factor structure of the Spanish version of the VQ with a large Spaniard sample $(N=846)$. Additionally, we analyzed the factorial equivalence of the VQ between the Spaniard sample and a Colombian sample $(\mathrm{N}=724)$ with similar 
The results showed that the VQ obtained good psychometric properties in the Spaniard

376

377

378

379

380

381

382

383

384

385

386

387

388

389

390

391

392

393

394

395

396

397

sample. Regarding internal consistency, the VQ showed appropriate Cronbach's alpha and

McDonald's omega values for both Progress (.85) and Obstruction (.84). These values are in the range of previous validation studies (e.g., Barrett et al., 2019; Ruiz et al., 2021; Smout et al., 2014). The two-factor model of the VQ obtained a good fit to the data. The fit of this model was very similar to the two-factor model with correlated error terms in Items 5 and 7, which was the factor model considered as more appropriate in the original validation study (Smout et al., 2014).

As in Ruiz et al. (2021), we chose the two-factor model due to its greater parsimony.

Additionally, the measurement model of the VQ showed adequate construct reliability and convergent and discriminant validity.

The VQ also showed measurement invariance at metric, scalar, and strict levels across countries and gender. The correlations of the VQ with other related instruments were theoretically coherent and equivalent to those found in other studies (Ruiz et al., 2021; Smout et al., 2014). Specifically, VQ-Progress was positively correlated with life satisfaction and negatively with emotional symptoms, experiential avoidance, and cognitive fusion. As expected, the opposite pattern of correlations was found between VQ-Obstruction and the constructs mentioned above. Thus, these results add further empirical evidence of the adaptive role of valued living according to the ACT model.

The Spanish version of the VQ showed similar psychometric properties in this Spaniard sample to the previous study with Colombian samples (Ruiz et al., 2021). Accordingly, this study presents further evidence of the robust psychometric properties of the VQ (e.g., Barrett et al., 2019; Reilly et al., 2019). To our best knowledge, this study presents the first analysis of factorial equivalence of the VQ across different cultures. The evidence of scalar invariance is 
398 relevant because it permits comparing scores across Spaniard and Colombian samples (Greiff \&

399 Scherer, 2018). In this regard, the Spaniard and Colombian samples analyzed in this study

400 showed similar mean scores on the VQ subscales, which preliminarily indicates that there seems

401 to be similar levels of valued living in these countries. Further studies might compare valued

402 living scores across larger Spaniard and Colombian samples This study also adds evidence of the

403 factorial equivalence of the Spanish version of the VQ across gender. As in Ruiz et al. (2021),

404 the VQ mean scores did not differ across gender. Thus, it seems that valued living does not differ

405 across gender in these two Spanish-speaking countries.

406 It is worth mentioning some of the limitations of this study. Firstly, the VQ was not

407 administered to a clinical sample. Further studies should analyze the psychometric properties of

408 the VQ in Spaniard clinical participants and the measurement invariance across clinical and

409 nonclinical individuals. Given that the study conducted by Ruiz et al. (2021) found strict

410 measurement invariance of the VQ across Colombian clinical and nonclinical participants, we

411 would expect to find similar results in Spaniard samples. Secondly, the percentage of women

412 was significantly higher than the percentage of men in the Spaniard sample. However, this

413 limitation was reduced by the results obtained in the measurement invariance across gender.

414 Thirdly, the VQ was only correlated with other self-reports, which could have inflated the

415 correlations found among the VQ subscales and the other instruments. Fourthly, the participants'

416 mean age was relatively low, which might be a consequence of the more frequent use of the

417 internet and social media by young people in Spain. Further studies should explore the

418 psychometric properties of the VQ in samples of older participants. Lastly, we did not test the

419 treatment sensitivity of the VQ in Spaniard samples. Subsequent studies should analyze this

420 issue. It would be expected that the VQ would show treatment sensitivity in Spaniard samples 
421 according to previous research that used the Spanish version of the VQ in Colombia (e.g., Ruiz 422 et al., 2018; Ruiz, Peña-Vargas, et al., 2020).

423 Despite these limitations, the current study has some practical implications for Spaniard 424 researchers and mental health professionals. Firstly, the VQ instrument might be used in several 425 research contexts, such as in survey studies that analyze the role of values in mental health and 426 clinical studies analyzing the efficacy and processes of change in ACT interventions. Secondly, 427 the VQ can be adopted in the routine assessment conducted by ACT practitioners. Lastly, the VQ 428 can be used by researchers that aim to compare valued living across gender and Spain and 429 Colombia.

430

\section{Conclusions}

The findings of this study are consistent with the previous analysis of the psychometric properties of the Spanish version of the VQ in Colombian samples (Ruiz et al., 2021). The current study adds empirical evidence of the good fit of the two-factor model of the VQ and its measurement invariance across Spaniard and Colombian samples. The factorial equivalence of the Spanish version of the VQ permitted comparing its scores across these countries. Further studies might analyze the measurement invariance of the VQ across additional Spanish-speaking countries and across different languages.

In conclusion, the VQ showed to be a reliable and valid instrument to measure valued

440 living according to the ACT model in a large Spaniard sample.

441

442 Acknowledgments

443

444

445 References 
446 Atienza, F. L., Pons, D., Balaguer, I., \& García-Merita, M. (2000). Propiedades psicométricas de 447 la Escala de Satisfacción con la Vida en adolescentes [Psychometric properties of the 448 Satisfaction with Life Scale in adolescents]. Psicothema, 12, 314-319.

449 Ato, M., López-García, J. J., \& Benavente, A. (2013). A classification system for research 450 designs in psychology. Annals of Psychology, 29, 1038-1059.

451 Barrett, K., O'Connor, M., \& McHugh, L. (2019). A Systematic Review of Values-Based 452 Psychometric Tools Within Acceptance and Commitment Therapy (ACT). The 453 Psychological Record, 69, 457-485.

454

455

Bond, F. W., Hayes, S. C., Baer, R. A., Carpenter, K. M., Guenole, N., Orcutt, H. K., ... Zettle, R. D. (2011). Preliminary psychometric properties of the Acceptance and Action Questionnaire - II: A revised measure of psychological inflexibility and experiential avoidance. Behavior Therapy, 42, 676-688.

Brown, T. A. (2015). Confirmatory factor analysis for applied research. NY: Guilford publications.

Cano-García, F. J., \& Rodríguez-Franco, L. (2002). Evaluación del lenguaje interno ansiógeno y depresógeno en la experiencia de dolor crónico [Assessment of anxious and depressive self-talk in chronic pain experience]. Apuntes de Psicología, 20, 329-346.

Carvalho, S. A., Palmeira, L., Pinto-Gouveia, J., Gillanders, D., \& Castilho, P. (2018). The utility of the valuing questionnaire in chronic pain. Journal of Contextual Behavioral Science, 9, 21-29. https://doi.org/10.1016/j.jcbs.2018.06.002

Chen, F. F. (2007). Sensitivity of goodness of fit indexes to lack of measurement invariance. Structural Equation Modeling: A Multidisciplinary Journal, 14, 464-504. 
468 Cheung, G. W., \& Rensvold, R. B. (2002). Evaluating goodness-of-fit indexes for testing

469

470

471

472

473

474

475

476

477

478

479

480

481

482

483

484

485

486

487

488

489

measurement invariance. Structural Equation Modeling: A Multidisciplinary Journal, 9, $233-255$.

Daza, P., Novy, D. M., Stanley, M., \& Averill, P. (2002). The Depression Anxiety Stress Scale21: Spanish translation and validation with a Hispanic sample. Journal of Psychopathology and Behavioral Assessment, 24, 195-205.

Diener, E., Emmons, R. A., Larsen, R. J., \& Griffin, S. (1985). The Satisfaction with Life Scale. Journal of Personality Assessment, 49, 71-75.

Forman, E. M., Herbert, J. D., Moitra, E., Yeomans, P. D., \& Geller, P. A. (2007). A randomized controlled effectiveness trial of Acceptance and Commitment Therapy and Cognitive Therapy for anxiety and depression. Behavior Modification, 31(6), 772-799.

Fornell, C., \& Larcker, D. F. (1981). Evaluating structural equation models with unobservable variables and measurement error. Journal of Marketing Research, 18, 39-50.

Gaudiano, B. A., \& Herbert, J. D. (2006). Acute treatment of inpatients with psychotic symptoms using Acceptance and Commitment Therapy: Pilot results. Behaviour Research \& Therapy, 44(3), 415-437.

Gillanders, D. T., Bolderston, H., Bond, F. W., Dempster, M., Flaxman, P. E., Campbell, ... Remington, B. (2014). The development and initial validation of the Cognitive Fusion Questionnaire. Behavior Therapy, 45, 83-101.

Gil, E., Luciano, C., Ruiz, F. J. y Valdivia-Salas, S. (2012). A preliminary demonstration of transformation of functions through hierarchical relations. International Journal of Psychology and Psychological Therapy, 12, 1-20. 
490 Gil-Luciano, B., Calderón-Hurtado, T., Tovar, D., Sebastián, B., \& Ruiz, F. J. (2019). How are 491 triggers for repetitive negative thinking organized? A relational frame analysis.

$492 \quad$ Psicothema, 31, 53-59.

493 Greiff, S., \& Scherer, R. (2018). Still comparing apples with oranges? Some thoughts on the 494 principles and practices of measurement invariance testing. European Journal of Psychological Assessment, 34, 141-144.

496

497

498

499

500

501

502

503

504

505

506

507

508

509

510

511

Hayes, S. C., Barnes-Holmes, D., \& Roche, B. (Eds.) (2001). Relational Frame Theory: A PostSkinnerian account of human language and cognition. New York: Plenum Press.

Hayes, S. C., Luoma, J. B., Bond, F. W., Masuda, A., \& Lillis, J. (2006). Acceptance and commitment therapy: Model, processes and outcomes. Behaviour Research and Therapy, $44,1-25$.

Hayes, S. C., Strosahl, K. D., \& Wilson, K. G. (1999). Acceptance and commitment therapy. An experiential approach to behavior change. New York: Guilford.

Hayes, S. C., Strosahl, K. D., \& Wilson, K. G. (2011). Acceptance and commitment therapy: The process and practice of mindful change. New York, NY: Guilford Press.

Hollon, S. D., \& Kendall, P. C. (1980). Cognitive self-statements in depression: Development of an Automatic Thoughts Questionnaire. Cognitive Therapy and Research, 4, 383-395. http://dx.doi.org/10.1007/BF01178214

Hu, L., \& Bentler, P. M. (1999). Cutoff criteria for fit indexes in covariance structure analysis: Conventional criteria versus new alternatives. Structural Equation Modeling, 6, 1-55.

Jöreskog, K. G. (2005). Structural equation modeling with ordinal variables using LISREL. Technical report, Scientific Software International, Inc., Lincolnwood, IL. 
512 Jöreskog, K. G., \& Sörbom, D. (1999). LISREL 8.30. Chicago, IL: Scientific Software 513 International.

514 Kelley, K., \& Lai, K. (2012). MBESS: MBESS. R package version 3.3.2. Retrieved from $515 \quad$ http://CRAN.R-project.org/package=MBESS.

516 Kelley, K., \& Pornprasertmanit, S. (2016). Confidence intervals for population reliability 517 coefficients: Evaluation of methods, recommendations, and software for composite 518 measures. Psychological Methods, 21, 69-92. http://dx.doi.org/10.1037/a0040086

519 Kenny, D. A., Kaniskan, B., \& McCoach, D. B. (2015). The performance of RMSEA in models 520 with small degrees of freedom. Social Methods \& Research, 44, 486-507.

521 https://doi.org/10.1177/0049124114543236

522 Kline, R. B. (2005). Principles and practice of structural equation modeling ( $2^{\text {nd }}$ ed.). New 523 York: Guilford Press.

524 Kline, R. B. (2016). Principles and practice of structural equation modeling (4th ed.). New York: $525 \quad$ Guilford Press.

526 Lenhard, W., \& Lenhard, A. (2016). Calculation of effect sizes. Retrieved from:

527 https://www.psychometrica.de/effect_size.html. Dettelbach, Germany: Psychometrica.

528 Levin, M. E., Krafft, J., Hicks, E. T., Pierce, B. \& Twohig, M. P. (2020). A randomized

529

530

531

532

533

534 dismantling trial of the open and engaged components of acceptance and commitment therapy in an online intervention for distressed college students. Behavior Research and Therapy, 126,103557

Lovibond, P. F., \& Lovibond, S. H. (1995). The structure of negative emotional states: Comparison of the Depression Anxiety Stress Scales (DASS) with the Beck Depression and Anxiety Inventories. Behaviour Research and Therapy, 33, 335-343. 
535 Luciano, C., Valdivia-Salas, S., \& Ruiz, F. J. (2012). The self as the context for rule-governed 536 behavior. In L. McHugh, \& I. Stewart (Eds.), The self and perspective taking: Research and applications (pp. 143-160). Oakland, CA: Context Press.

538 Lundgren, T., Luoma, J. B., Dahl, J., Strosahl, K. D., \& Melin, L. (2012). The Bull's-Eye Values 539 Survey: A psychometric evaluation. Cognitive and Behavioral Practice, 19, 518-526.

540

541

542

543

544

545

546

547

548

549

550

551

552

553

554

555

556 measures. Multivariate Behavioral Research, 39, 479-515.

Mokkink, L. B., De Vet, H. C., Prinsen, C. A., Patrick, D. L., Alonso, J., Bouter, L. M., \& Terwee, C. B. (2018). COSMIN Risk of Bias checklist for systematic reviews of patientreported outcome measures. Quality of Life Research, 27, 1171-1179.

Netemeyer, R. G., Williamson, D. A., Burton, S., Biswas, D., Jindal, S., Landreth, S., ... Primeaux, S. (2002). Psychometric properties of shortened versions of the Automatic Thoughts Questionnaire. Educational and Psychological Measurement, 62, 111-129.

Nunnally, J. C., \& Bernstein, I. H. (1994). Psychometric theory (3rd ed.). NY: McGraw-Hill. O'Connor, M., Whelan, R., Bricker, J., \& McHugh, L. (2020). Randomized controlled trial of a smartphone application as an adjunct to Acceptance and Commitment Therapy for smoking cessation. Behavior Therapy, 51, 162-177.

Reilly, E. D., Ritzert, T. R., Scoglio, A. A., Mote, J., Fukuda, S. D., Ahern, M. E., \& Kelly, M. M. (2019). A systematic review of values measures in acceptance and commitment therapy research. Journal of Contextual Behavioral Science, 12, 290-304.

Rickardsson, J., Zetterqvist, V., Kemani, M. K., Holmström, L., Andersson, E., \& Wicksell, R. K. (2019). Assessing values-Psychometric properties of the Swedish version of the 
557

558

559

560

561

562

563

564

565

566

567

568

569

570

571

572

573

574

575

576

577

578

579

Valuing Questionnaire in adults with chronic pain. Journal of Contextual Behavioral Science, 14, 40-49. https://doi.org/10.1016/j.jcbs.2019.08.009

Ruiz, F. J. (2020). The verbal nature of human joy and suffering [Blog entry]. ABAI Symbolic Language and Thought Blog. Retrieved from https://science.abainternational.org/theverbal-nature-of-human-joy-and-suffering/louise-mchughucd-ie/

Ruiz, F. J., García-Martín, M. B., Suárez-Falcón, J. C., \& Odriozola-González, P. (2017). The hierarchical factor structure of the Spanish version of Depression Anxiety and Stress Scale-21. International Journal of Psychology and Psychological Therapy, 17, 97-105.

Ruiz, F. J., Flórez, C. L., García-Martín, M. B., Monroy-Cifuentes, A., Barreto-Montero, K., García-Beltrán, D. M., ... Gil-Luciano, B. (2018). A multiple-baseline evaluation of a brief acceptance and commitment therapy protocol focused on repetitive negative thinking for moderate emotional disorders. Journal of Contextual Behavioral Science, 9, $1-14$.

Ruiz, F. J., Luciano, C., Flórez, C. L., Suárez-Falcón, J. C., \& Cardona-Betancourt, V. (2020). A Multiple-Baseline Evaluation of Acceptance and Commitment Therapy Focused on Repetitive Negative Thinking for Comorbid Generalized Anxiety Disorder and Depression. Frontiers in Psychology, 11, 356. https://doi.org/10.3389/fpsyg.2020.00356

Ruiz, F. J., Langer, A. I., Luciano, C., Cangas, A. J., \& Beltrán, I. (2013). Measuring experiential avoidance and psychological inflexibility: The Spanish translation of the Acceptance and Action Questionnaire. Psicothema, 25, 123-129.

Ruiz, F. J., Peña-Vargas, A., Ramírez, E. S., Suárez-Falcón, J. C., García-Martín, M. B., GarcíaBeltrán, D. M., ... Sánchez, P. D. (2020). Efficacy of a two-session repetitive negative thinking-focused acceptance and commitment therapy (ACT) protocol for depression and 
580

581

582

583

584

585

586

587

588

589

590

591

592

593

594

595

596

597

598

599

600

generalized anxiety disorder: A randomized waitlist control trial. Psychotherapy, 57, 444456.

Ruiz, F. J., Segura-Vargas, M. A., Odriozola-González, P. \& Suárez-Falcón, J. C. (2020).

Psychometric properties of the Automatic Thoughts Questionnaire-8 in Spain. PeerJ, 8, e9747.

Ruiz, F. J., Suárez-Falcón, J. C., Segura-Vargas, M.A., \& Gil-Luciano, B. (2021). Psychometric properties of the Spanish version of the Valuing Questionnaire in Colombian clinical and nonclinical samples (manuscript submitted for publication).

Ruiz, F. J., Suárez-Falcón, J. C., Flórez, C. L., Odriozola-González, P., Tovar, D., LópezGonzález, S., \& Baeza-Martín, R. (2019). Validity of the Satisfaction with Life Scale in Colombia and factorial equivalence with Spanish data. Revista Latinoamericana de Psicología, 51, 58-65.

Ruiz, F. J., Suárez-Falcón, J. C., Riaño-Hernández, D., \& Gillanders, D. (2017). Psychometric properties of the Cognitive Fusion Questionnaire in Colombia. Revista Latinoamericana de Psicología, 49, 80-87.

Smout, M., Davies, M., Burns, N., \& Christie, A. (2014). Development of the Valuing Questionnaire (VQ). Journal of Contextual Behavioral Science, 3, 164-172.

Trompetter, H. R., ten Klooster, P. M., Schreurs, K. M. G., Fledderus, M., Westerhof, G. J., \& Bohlmeijer, E. T. (2013). Measuring values and committed action with the Engaged Living Scale (ELS): Psychometric evaluation in a nonclinical sample and a chronic pain sample. Psychological Assessment, 25(4), 1235-1246. 
601 Veehof, M. M., Oskam, M., Schreurs, K. M. G., \& Bohlmeijer, E. T. (2011). Acceptance-based 602 interventions for the treatment of chronic pain: A systematic review and meta603 analysis. Pain, 152, 533-542.

604 Vilardaga, R., Hayes, S. C., Levin, M. E., \& Muto, T. (2009). Creating a strategy for progress: A 605 contextual behavioral science approach. The Behavior Analyst, 32, 105-133.

606 Wilson, K. G., \& DuFrene, T. (2009). Mindfulness for two: An acceptance and commitment 607 therapy approach to mindfulness in psychotherapy. Oakland, CA: New Harbinger.

608 Wilson, K. G., Sandoz, E. K., Kitchens, J., \& Roberts, M. (2010). The Valued Living

609 Questionnaire: Defining and measuring valued action within a behavioral framework. The $610 \quad$ Psychological Record, 60, 249-272. 


\section{Table $\mathbf{1}$ (on next page)}

Goodness-of-Fit Indexes of the Two-Factor Model and the Two-Factor Model with Error Terms Allowed to Correlate for Items 5 and 7 in Sample 1 


\section{$1 \quad$ Table 1}

2 Goodness-of-Fit Indexes of the Two-Factor Model and the Two-Factor Model with Error Terms

3 Allowed to Correlate for Items 5 and 7 in Sample 1

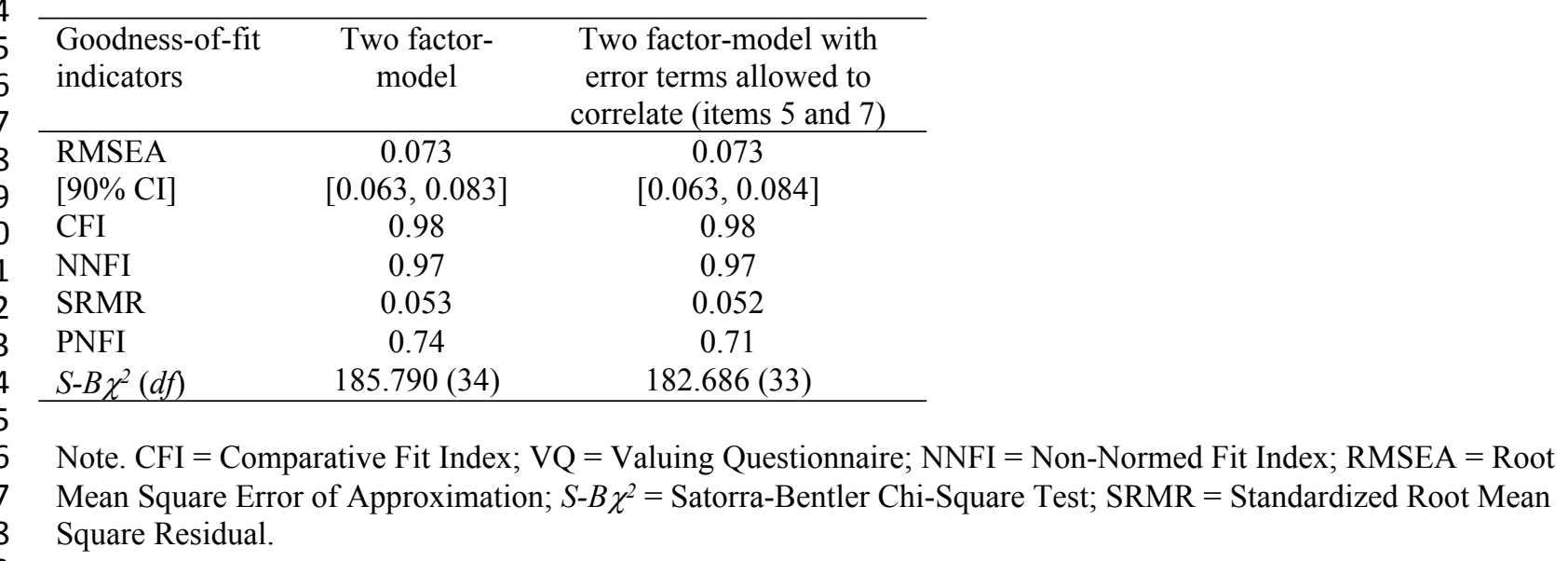




\section{Table 2 (on next page)}

Results of the Metric and Scalar Invariance Analyses Across Spain and Colombia and Gender 
1 Table 2

2 Results of the Metric and Scalar Invariance Analyses Across Spain and Colombia and Gender

3

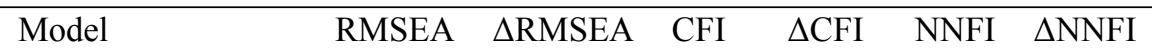

Measurement invariance across countries

\begin{tabular}{llllccc} 
MG Baseline model & 0.075 & & 0.976 & & 0.969 & \\
Metric invariance & 0.071 & 0.004 & 0.976 & 0.000 & 0.972 & 0.003 \\
Scalar invariance & 0.070 & 0.001 & 0.974 & -0.002 & 0.972 & 0.000 \\
Strict invariance & 0.065 & 0.005 & 0.975 & 0.001 & 0.976 & 0.004 \\
\hline
\end{tabular}

Measurement invariance across gender

$\begin{array}{lllllll}\text { Metric invariance } & 0.071 & 0.004 & 0.976 & 0.000 & 0.972 & 0.003\end{array}$

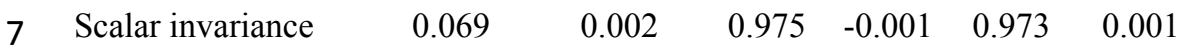

\begin{tabular}{lllllll} 
Strict invariance & 0.065 & 0.004 & 0.975 & 0.000 & 0.976 & 0.003 \\
\hline
\end{tabular}

8

9

10

11

12

13

14

15

16

17

18

19

20

21

Peer) reviewing PDF | (2021:04:60786:1:1:NEW 9 Nov 2021) 


\section{Table 3 (on next page)}

Item Description and Corrected Item-Total Correlations in Sample 1 
$1 \quad$ Table 3

2 Item Description and Corrected Item-Total Correlations in Sample 1

\begin{tabular}{|c|c|}
\hline Item number and description & $\begin{array}{l}\text { Corrected item- } \\
\text { total correlations }\end{array}$ \\
\hline $\begin{array}{l}\text { 1. Pasé un montón de tiempo pensando sobre el pasado o el futuro en } \\
\text { vez de dedicarme a actividades que eran importantes para mí [I spent a } \\
\text { lot of time thinking about the past or future, rather than being engaged in } \\
\text { activities that mattered to me]. OBSTRUCTION }\end{array}$ & .69 \\
\hline $\begin{array}{l}\text { 2. Estuve básicamente en "piloto automático" la mayor parte del tiempo } \\
\text { [I was basically on "auto-pilot" most of the time]. OBSTRUCTION }\end{array}$ & .70 \\
\hline $\begin{array}{l}\text { 3. Trabajé para conseguir mis metas incluso cuando no me sentía } \\
\text { motivado [I worked toward my goals even if I didn't feel motivated to]. } \\
\text { PROGRESS }\end{array}$ & .52 \\
\hline $\begin{array}{l}\text { 4. Estuve orgulloso de cómo viví mi vida [I was proud about how I } \\
\text { lived my life]. PROGRESS }\end{array}$ & .71 \\
\hline $\begin{array}{l}\text { 5. Hice progresos en las áreas de mi vida que más me importan [I made } \\
\text { progress in the areas of my life I care most about]. PROGRESS }\end{array}$ & .71 \\
\hline $\begin{array}{l}\text { 6. Los pensamientos, sentimientos y recuerdos difíciles se interpusieron } \\
\text { en el camino de lo que quería hacer. [Difficult thoughts, feelings or } \\
\text { memories got in the way of what I really wanted to do]. } \\
\text { OBSTRUCTION }\end{array}$ & .62 \\
\hline $\begin{array}{l}\text { 7. Continué mejorando en ser el tipo de persona que deseo ser [I } \\
\text { continued to get better at being the kind of person I want to be]. } \\
\text { PROGRESS }\end{array}$ & .71 \\
\hline $\begin{array}{l}\text { 8. Cuando las cosas no fueron según lo planeado, me di por vencido } \\
\text { fácilmente [When things didn't go according to plan, I gave up easily]. } \\
\text { OBSTRUCTION }\end{array}$ & .51 \\
\hline $\begin{array}{l}\text { 9. Me sentí como si tuviera un propósito en la vida [I felt like I had a } \\
\text { purpose in life]. PROGRESS }\end{array}$ & .62 \\
\hline $\begin{array}{l}\text { 10. Parecía como si estuviera comportándome de manera mecánica en } \\
\text { vez de centrarme en lo que era importante para mí [It seemed like I was } \\
\text { just "going through the motions" rather than focusing on what was } \\
\text { important to me]. OBSTRUCTION }\end{array}$ & .69 \\
\hline
\end{tabular}

3 


\section{Table 4 (on next page)}

Pearson Correlations between the VQ Scores and Other Relevant Self-Report Measures in Sample 1 


\section{$1 \quad$ Table 4}

2 Pearson Correlations between the VQ Scores and Other Relevant Self-Report Measures in

3 Sample 1

\begin{tabular}{lcc}
\hline Measure & $\begin{array}{c}r \text { with } \\
\text { Progress }\end{array}$ & $\begin{array}{c}r \text { with } \\
\text { Obstruction }\end{array}$ \\
\hline VQ-Obstruction & $-.51^{*}$ & \\
AAQ-II (Experiential avoidance) & $-.49^{*}$ & $.66^{*}$ \\
CFQ (Cognitive fusion) & $-.44^{*}$ & $.68^{*}$ \\
DASS-Total & $-.36^{*}$ & $.65^{*}$ \\
DASS-21 - Depression & $-.48^{*}$ & $.66^{*}$ \\
DASS-21 - Anxiety & $-.23^{*}$ & $.53^{*}$ \\
DASS-21 - Stress & $-.24^{*}$ & $.54^{*}$ \\
SWLS (Life satisfaction) & $.64^{*}$ & $-.53^{*}$ \\
\hline
\end{tabular}

4 Note. AAQ-II: Acceptance and Action Questionnaire - II; DASS - 21: Depression, Anxiety, and Stress Scales - 21;

5 SWLS: Satisfaction with Life Scale; $\mathrm{VQ}=$ Valuing Questionnaire. ${ }^{*} p<.001$

6 
Figure 1

Completely standardized solution for the two-factor model of the Valuing Questionnaire in Sample 1.

Figure 1. Completely standardized solution for the two-factor model of the Valuing Questionnaire in Sample 1.

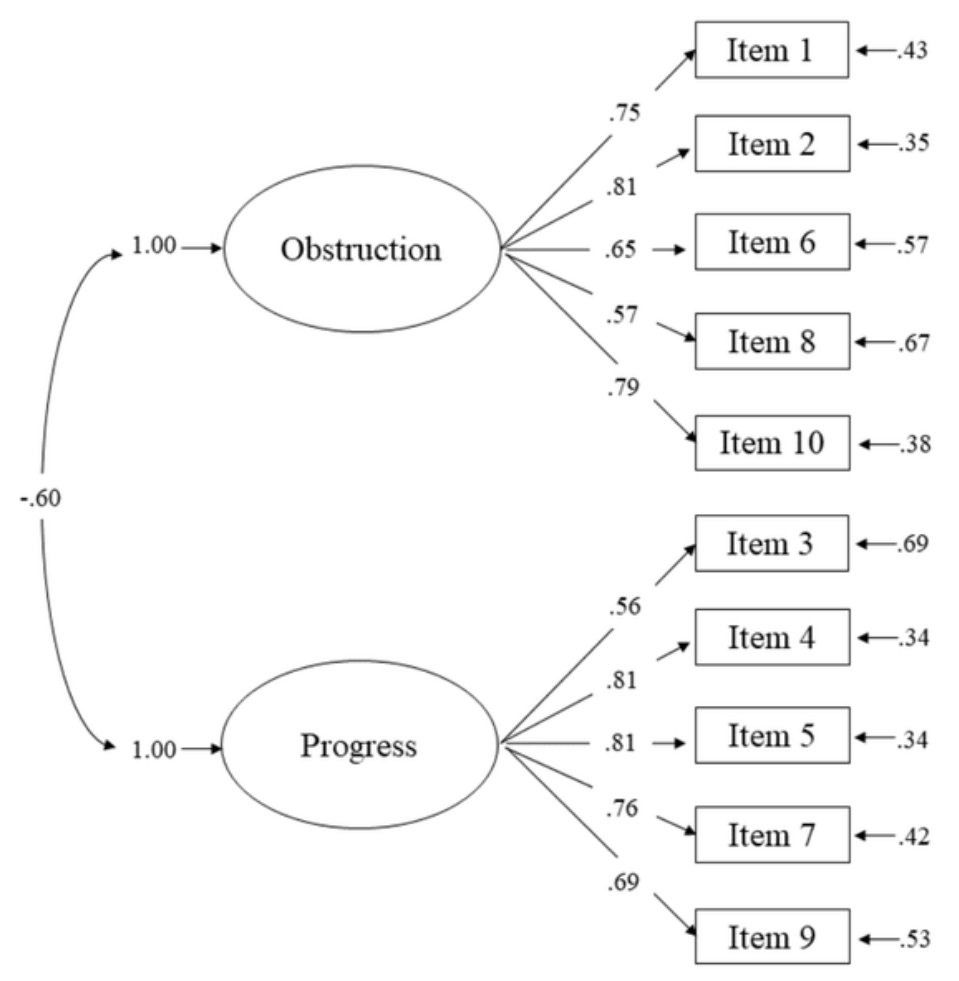

